\title{
Modelo Dinâmico Inercial Rígido para \\ Escoamento Transitório Lento em Sistemas de Abastecimento de Água.
}

\section{Inertial Rigid Dynamic Model for Slow Transient Flow in Water Supply Systems}

Luiz Fernando Resende dos Santos Anjo'; Edevar Luvizotto Júnior ${ }^{2}$ e Stênio de Sousa Venâncio ${ }^{3}$

${ }^{1}$ Professor, Departamento de Engenharia Civil, Universidade Federal do Triângulo Mineiro - Uberaba, Minas Gerais, Brasil luizfernando@civil.uftm.edu.br

\footnotetext{
${ }^{2}$ Professor, Departamento de Recursos Hídricos - Engenharia Civil, Arquitetura e Urbanismo, Universidade Estadual de Campinas - Cidade Universitária Zeferino Vaz, Barão Geraldo, Campinas, São Paulo, Brasil edevar@fec.unicamp.br
}

${ }^{3}$ Professor, Departamento de Engenharia Civil, Universidade Federal do Triângulo Mineiro - Uberaba, Minas Gerais, Brasil stenio@civil.uftm.edu.br

\section{RESUMO}

Este texto descreve as etapas para a utilização da estrutura originalmente proposta por Todini e Pilati (1998), no chamado método gradiente (MG). Este método é utilizado paraanálise em regime permanente em instalações com condutos forçados, formulando um modelo dinâmico inercial rígido (MDIR), para a avaliação dosescoamentos transitórios lentos. Para tanto, as bases teóricas para esta nova modelação, justificadas através do equacionamento geral do escoamento fluido em condutos forçados, são apresentadas. Os resultados obtidos pelo MDIR são comparados com os resultados obtidos pelo programa EPANET (Agência Nacional de Proteção Ambiental Norte Americana) que utiliza o método gradiente, com o intuito de demonstrar o efeito da inércia. As discussões sobre a importância de incorporar o efeito de inércia são apresentados por meio de estudos de caso. Os resultados demonstram as vantagens do modelo proposto na formulação não inercial na análise de manobras lentas. Desde que a estrutura global do MG seja preservada, o novo método pode ser facilmente implementado em modelos baseados neste método. Na sua forma original, a convergência numérica é devido ao processo de minimização de resíduos: no MDIR, a convergência evolui com o tempo (convergência no tempo) em que o fenómeno é descrito fisicamente de uma condição inicial para qualquer outra condição.

Palavras Chave: Método gradiente. Transitórios lentos. Modelo dinâmico inercial rígido

\begin{abstract}
This text describes the stages for the use of the structure originally proposed by Todini and Pilati (1998), in the so-called gradient method (GM). The method is used for analysis in a steady state regime in facilities with penstocks fomulating an inertial rigid dynamic model (MDIR-modelo dinâmico inercial rígido), to evaluate the slow transient flows. For this purpose the theoretical bases for this new modeling, justified by the general examination of liquid flow in penstocks are presented. The results obtained using MDIR are compared to the results obtained by the EPANET program (U S Environmental Protection Agency, which uses the gradient method to demonstrate the effect of inertia. The discussions about the importance of incorporating the effect of inertia are shown in case studies. The results demonstrate the advantages of the model proposed for the non-inertial formulation in the analysis of slow maneuvers. As long as the global structure of the GM is preserved, the new method can easily be implemented in models based on this method. In its original form the numerical convergence is due to the process of minimizing wastes in the MDIR, convergence evolves over the time (convergence in time) in which the phenomenon is described physically from an initial condition to any other condition. .
\end{abstract}

Keywords: Gradient method. Slow transients. Inertia rigid dynamic model 


\section{INTRODUÇÃO}

$\mathrm{Na}$ operação real de qualquer sistema de abastecimento de água é inerente a variação com o tempo das variáveis hidráulicas. Assim, em modelagem, torna-se necessário conhecer o comportamento da instalação devido à ocorrência do regime transiente, gerado por manobras operacionais (abrir/fechar válvula, ligar/desligar bomba, etc.). Deve-se deixar claro que essas manobras podem ser normais ou acidentais, sendo que para qualquer uma delas o sistema deve responder adequadamente.

A análise do escoamento em condutos forçados, em sistemas hidráulicos, é usualmente considerada unidimensional, segundo o eixo dos condutos, seguindo diferentes hipóteses simplificadoras, que permitem a concepção de distintos modelos. Estes modelos são classificados, como observa Cabrera, Garcia-Serra e Iglesias (1994), em modelos dinâmicos e estáticos, segundo a variação ou não do escoamento no tempo. Os modelos dinâmicos se subdividem em modelos dinâmicos inerciais e não inerciais, caso levem ou não a inércia como elemento na variação temporal. Os modelos dinâmicos inerciais podem, por sua vez, considerar ou não efeitos de deformação do fluido e do conduto, como decorrência, o que leva a classificá-los em elásticos ou não elásticos (rígidos).

Como observação, baseada no parágrafo introdutório, para uma análise correta de comportamentos dinâmicos, o emprego do modelo dinâmico inercial elástico torna-se necessário. Entretanto, para sistemas muito complexos, esta abordagem pode ser bastante dispendiosa, uma vez que demanda muito tempo de processamento. Como simplificação (na maioria dos casos) um modelo dinâmico não inercial é aplicado.

Nesta linha, Rossman (1993), desenvolveu um modelo computacional, denominado EPANET, que se consagrou como ferramenta para a análise de redes de distribuição de água em regime permanente e em período extensivo. O programa emprega a solução do método gradiente nas simulações dos sistemas hidráulicos. Além da simulação hidráulica o modelo também incorpora a possibilidade de simulações para avaliar a qualidade da água, pela distribuição, no sistema, de um determinado componente (ex.: cloro), em período extensivo e com base num modelo dinâmico não inercial.

Iglesias (2004) chama a atenção para o fato que a analise em período extensivo, usando uma modelação estática, pode conduzir a erros de resultados pelo fato devido à negligência do efeito da inércia. Ele apresenta uma técnica para estabelecer qual modelo é mais adequado, para a simulação de um sistema hidráulico, definindo uma fronteira que separa a simulação em período extensivo com o modelo inercial rígido. Essa fronteira é determinada pela aplicação dos dois modelos num mesmo sistema hidráulico, onde se observa os erros gerados pela aplicação da simulação em período extensivo ao invés do modelo inercial rígido. Admite-se que a simulação em período extensivo é valida quando os erros relativos forem inferiores a 5\% dos resultados obtidos pela aplicação do modelo inercial rígido. Nas simulações são realizadas várias condições: variação de demanda ao longo do tempo, variação do expoente da curva de demanda, manobras em válvulas e bombas, etc.

Islam e Chaudhry (1998) fizeram uma análise da dis- tribuição espacial e temporal de componentes, todavia empregando uma modelação dinâmica inercial rígida. Comparando os resultados com os obtidos pelo EPANET concluíram que o efeito da inércia era relevante no caso da dispersão desse componente. Justificaram o modelo rígido como satisfatório desde que as manobras realizadas fossem lentas a ponto de não causarem a compressibilidade do fluido e a deformação dos tubos (denominando essa condição de transitório lento). Outros autores também já propuseram solução para esse tipo de escoamento. Entretanto, tais soluções parecem não serem de fácil emprego e, portanto, não sensibilizaram a comunidade técnico-científica.

Conclui-se que o modelo dinâmico inercial rígido, para a análise de sistemas de abastecimento de água, pode ser uma solução bastante interessante para se levar os efeitos de inércia do escoamento fluido embora, se saiba, que está se desprezando efeitos elásticos que podem ocorrer. Por outro lado o programa EPANET e os seus "derivados" comerciais apresentam a técnica mais empregada e difundida na avaliação estática e de período extensivo, o que o tornou quase um padrão e uma unanimidade na comunidade técnico-científica.

Em decorrência do que foi apresentado encontrou-se motivação para o desenvolvimento de uma pesquisa visando implementar o modelo dinâmico inercial rígido, com a proposta de aproveitar a estrutura do programa EPANET. Isto requer uma modificação na montagem do sistema de equações, que definem as condições de escoamento, para permitir a análise de transitórios lentos.

Acredita-se que com esse expediente, os profissionais de engenharia, que já empregam cotidianamente o software EPANET, serão beneficiados, no que tange a realização de simulações dinâmicas mais próximas da realidade.

\section{MODELO HIDRÁULICO PARA CONDUTOS FORÇADOS}

As equações hidrodinâmicas gerais, que governam o escoamento nos condutos forçados, permitem a determinação das variáveis de estado, carga e vazão ao longo da tubulação, no tempo. Sob as hipóteses do modelo dinâmico inercial elástico, estas equações podem ser expressas através das equações da continuidade e da quantidade de movimento, respectivamente, na forma da equação 1 (WYLIE; STREETER, 1993):

$$
\left\{\begin{array}{c}
\frac{g A}{a^{2}} \frac{\partial H}{\partial t}+\frac{\partial Q}{\partial x}=0 \\
\frac{\partial H}{\partial x}+\frac{1}{g A} \frac{\partial Q}{\partial t}+f \frac{Q|Q|}{2 g D A^{2}}=0
\end{array}\right.
$$

Sob a hipótese de modelo rígido a celeridade de propagação $(a)$ torna-se infinita, levando à zero o primeiro termo do lado esquerdo da equação da continuidade, reduzindo esta equação a $\partial \mathrm{Q} / \partial \mathrm{x}=0$. Daí conclui-se que a vazão será a mesma em toda a extensão da tubulação para cada instante de tempo . 
$t$ Logo, $Q=Q(t)$ e $\partial Q / \partial t=d Q / d t$.

A equação da quantidade de movimento integrada entre as seções extremas da tubulação, (1) seção de montante e (2) seção de jusante, distantes um comprimento $L$, medido ao longo de seu eixo, permite obter a equação 2 :

$$
\left(H_{2}-H_{1}\right)+f \frac{L Q|Q|}{2 g D A^{2}}=-\frac{L}{g A} \frac{d Q}{d t}
$$

que é a equação que governa o modelo dinâmico inercial rígido. Se as variações de vazão no tempo deixam de existir; $d Q / d t=0$, representando a condição de regime permanente, leva-se à equação 3 :

$$
\left(H_{2}-H_{1}\right)+f \frac{L Q|Q|}{2 g D A^{2}}=0 .
$$

As equações (1), (2) e (3), mostram a hierarquia entre as diversas concepções, resultante das simplificações admitidas durante a elaboração dos modelos. A essência deste trabalho está em aproveitar a estrutura de uma modelação estática, baseada em (3), para uma modelação dinâmica, baseada em (2), permitido simultaneamente análises dinâmicas e estáticas.

\section{MODELO ORIGINAL}

Dada uma rede hidráulica, por condutos forçados, definida por sua topologia, características físicas das tubulações e restrições (tais como consumos nodais, nós com cargas fixas e uma lei de variação de carga para cada tubo $i$, expressa por $\mathrm{f}(\mathrm{Qi})$ ). Todini e Pilati (1988) apresentam, na forma matricial, o problema da determinação da vazão, pelos tubos e cargas nos nós, assumindo condição de regime permanente como mostra a equação 4:

$$
\left\{\begin{array}{c}
A_{12} H+f(Q)=-A_{10} H_{0} \\
A_{21} Q=q
\end{array},\right.
$$

sendo;

$\mathrm{A}_{12}=\mathrm{A}_{21}{ }^{\mathrm{T}}$ (np x np) - matriz de incidência de incógnitas cargas nodais.

$\mathrm{A}^{10}$ (np x no) matriz de incidência de nós com carga fixa.

$\mathrm{Q}^{\mathrm{T}}=\left\{\mathrm{Q}_{1}, \ldots, \mathrm{Q}_{\mathrm{p}}\right\}(1 \times \mathrm{np})$ - vazão incógnita em cada tubo.

$\mathrm{H}^{\mathrm{T}}=\left\{\mathrm{H}_{1}, \ldots, \mathrm{H}_{\mathrm{p}}\right\}(1 \times \mathrm{x}$ no $)$ - cargas nodais incógnitas.

$\mathrm{q}^{\mathrm{T}}=\left\{\mathrm{q}_{1}, \ldots, \mathrm{q}_{\mathrm{nn}}\right\}(1 \times \mathrm{nn})$ - demandas nodais conhecidas

$\mathrm{Ho}^{\mathrm{T}}=\left\{\mathrm{Ho}_{1}, \ldots, \mathrm{H}_{\mathrm{o}}{ }^{\mathrm{no}}\right\}(1 \times \mathrm{x}$ no $)$ - nós com cargas estabelecidas

(conhecidas ou fixas)

$\mathrm{f}(\mathrm{Q})^{\mathrm{T}}=\left\{\mathrm{f}\left(\mathrm{Q}_{1}\right), \ldots, \mathrm{f}\left(\mathrm{Q}_{\mathrm{np}}\right)\right\}$ - lei que expressa a variação de carga nos tubos.

com:

$\mathrm{nn}=$ número de nós com carga incógnita.

no = número de nós com carga fixa.

$\mathrm{np}=$ número de tubos com incógnita vazão.

Os elementos da matriz $A_{12}$ são escritos na forma:
$A_{12}(i, j)=\left\{\begin{aligned} 1 & \text { se a vazão no tubo i chega ao nó } \mathrm{j} \\ 0 & \text { se o tubo i e o nó j não estão conectados } \\ -1 & \text { se a vazão no tubo i sai do nó } \mathrm{j}\end{aligned}\right.$

e os elementos de $A_{10}$, definidos de forma similar a $A_{12}$, mas em relação aos nós com carga fixa.

Segundo Todini e Pilat (1988), o sistema representado na equação (4) pode ter mais de uma solução, dependendo do perfil da função $f\left(Q_{i}\right)$, mas sendo possível provar que, se todas as $f\left(Q_{i}\right)$ são funções monotomicamente crescentes, a solução do sistema de equações dado por (4) existe e é única.

Assumindo para cada tubo i, a função $f\left(Q_{i}\right)$ como:

$$
f\left(Q_{i}\right)=R_{i}\left|Q_{i}\right|^{n-1} Q_{i}
$$

obtém-se o sistema de equações:

$$
\left[\begin{array}{cc}
A_{11} & A_{12} \\
A_{21} & 0
\end{array}\right] \cdot\left[\begin{array}{l}
Q \\
H
\end{array}\right]=\left[\begin{array}{c}
-A_{10} H_{0} \\
q
\end{array}\right],
$$

onde $A_{11}$ é a matriz quadrada diagonal dos coeficientes de energia $R_{i}\left|Q_{i}\right|^{n-1}$ :

O sistema de equações (6) é o mesmo de (1), sendo a solução do sistema de equações não lineares, dado por (3), única no espaço, de todas as incógnitas cargas e de vazão.

Os autores encaminham a solução do sistema de equações (6) através do método de Newton-Raphson. Diferenciando o sistema:

$$
\left[\begin{array}{cc}
N A_{11} & A_{12} \\
A_{21} & 0
\end{array}\right] \cdot\left[\begin{array}{l}
d Q \\
d H
\end{array}\right]=\left[\begin{array}{l}
d E \\
d q
\end{array}\right] .
$$

Considerando a fórmula universal de perda de carga pode-se definir:

$$
f\left(Q_{i}\right)=R_{i}\left|Q_{i}\right| Q_{i}
$$

com $R_{i}=f_{i} L_{i} /\left(2 g D_{i} A_{i}^{2}\right)$ é representando o termo de resistência, avaliada em cada tubo $i$.

Após operações aritméticas das equações anteriores encontra-se:

$$
A_{21} G^{-1} A_{12} H^{K+1}=q_{2}^{K}-A_{21} G^{-1} A_{11}^{K} Q^{K}-A_{21} G^{-1} A_{10} H_{0}
$$

onde: $G$ é uma matriz diagonal quadrada de ordem np:

$$
G=\left[\begin{array}{ccc}
2 R_{1}\left|Q_{n p}\right| & \cdots & 0 \\
\cdots & \cdots & \cdots \\
0 & \cdots & 2 R_{n p}\left|Q_{n p}\right|
\end{array}\right]
$$

chamando o produto de matrizes, que multiplica as cargas incógnitas do primeiro termo, de $J$, e o vetor resultante do segundo membro de $F$ :

$$
J H^{K+1}=F,
$$


onde a matriz se associa à matriz Jacobiana e pode ser escrita como:

$$
J_{i j}=\left\{\begin{array}{l}
i=j \Rightarrow J_{i j}=\sum_{K \in i} \frac{1}{G_{i K}} \\
i \neq j \Rightarrow J_{i j}=-\frac{1}{G_{i j}} .
\end{array}\right.
$$

$O$ vetor resultante $F$ pode ser escrito:

$$
F_{i}=\left(\sum_{K \in i} Q_{K}-D_{i}\right)+\sum_{K \in i} \frac{1}{G_{K}} R_{K} Q_{K}\left|Q_{K}\right|+\sum_{j \in i} \frac{H_{0 j}}{G_{K}} \operatorname{sgn}\left(Q_{K}\right)
$$

Uma vez obtida a solução para o sistema de equação, dada por (11), para a determinação do vetor de cargas, podemse obter as vazões pela equação:

$$
Q^{K+1}=Q^{K}-G^{-1} A_{11}^{K} Q^{K}-G^{-1} A_{10} H_{0}-G^{-1} A_{12} H^{K+1} .
$$

A equação anterior pode ser reescrita na forma explícita:

$$
Q_{i j}^{K+1}=Q_{i j}^{K}-\left[\frac{1}{G_{i j}}\left[A_{11}\right]_{i j} Q_{i j}^{K} \sin a l\left(Q_{i j}^{K}\right)\right]+\frac{1}{G_{i j}}\left(H_{i}^{K+1}-H_{j}^{K+1}\right) .
$$

A solução iterativa é feita com base na solução proposta em (11). Para o sistema de equações, baseado nas cargas nodais, os valores de carga obtidos permitem a determinação, através da equação (14), das vazões pelos tubos, num processo iterativo, até o estabelecimento da convergência adotada. Este procedimento numérico encontra-se apresentado de maneira simplificada no manual do usuário do programa EPANET.

\section{MODELO PROPOSTO}

A equação do modelo rígido pode ser expressa na seguinte forma matricial, aproveitando-se das definições das matrizes, anteriormente definidas, para o modelo estático:

$$
A_{12} H+A_{10} H_{0}+f(Q)=-\beta \frac{d Q}{d t},
$$

$\beta$ é uma matriz diagonal quadrada de ordem np, com o elemento $(i, i)$, formado pela relação obtida do tubo i, dada por $\beta_{i i}=L_{i} /\left(g A_{i}\right)$.

Utilizando uma aproximação simples para $d Q / d t$, na forma: $\left(Q-Q_{0}\right) / \Delta t, Q_{0}$ é avaliada em $K$, relativa ao instante $(t)$, e $Q$ avaliada em $(K+1)$, no instante $(t+\Delta t)$.

Considerando que na evolução no tempo o termo de atrito possa ser avaliado em relação ao instante t, pode-se reescrever a equação anterior como:

$$
A_{12} H+A_{10} H_{0}+G Q_{0}+B Q=0,
$$

onde e são matrizes diagonais quadradas de ordem np:

$$
\begin{aligned}
G & =\left[\begin{array}{ccc}
R_{1}\left|Q_{01}\right|-B_{1} & \cdots & 0 \\
\cdots & \cdots & \cdots \\
0 & \ldots & R_{n p}\left|Q_{0 n p}\right|-B_{n p}
\end{array}\right] ; \\
B & =\left[\begin{array}{ccc}
\frac{L_{1}}{g A_{1} \Delta t} & \cdots & 0 \\
\cdots & \cdots & \ldots \\
0 & \cdots & \frac{\ddot{L}_{n p}}{g A_{n p} \Delta t}
\end{array}\right]
\end{aligned}
$$

O sistema de equações a ser resolvido para a solução do problema será:

$$
\left\{\begin{array}{c}
A_{12} H+B Q=G Q_{0}-A_{10} H_{0} \\
A_{21} Q=q
\end{array}\right.
$$

que resulta na forma matricial:

$$
\left[\begin{array}{cc}
B & A_{12} \\
A_{21} & 0
\end{array}\right] \cdot\left[\begin{array}{c}
Q \\
H
\end{array}\right]=\left[\begin{array}{c}
G Q_{0}-A_{10} H_{0} \\
q
\end{array}\right],
$$

sendo linear e tendo como propriedade importante o fato da matriz dos coeficientes ser constante, ou seja, montada apenas uma vez durante o processo de solução. O vetor do sistema é variável no tempo e dependerá da equação de resistência utilizada. Considerando a Fórmula Universal de perda de carga pode-se escrever:

$$
A_{21} B^{-1} A_{12} H=A_{21} B^{-1} G Q_{0}-A_{21} B^{-1} A_{10} H_{0}-q .
$$

Chamando o produto de matrizes, que multiplica as cargas incógnitas, de $J$, e o vetor resultante do segundo membro de $F$ :

$$
J H=F \quad,
$$

tem-se novamente um sistema, que é análogo ao do modelo estático anteriormente apresentado:

$$
J_{i j}=\left\{\begin{array}{l}
i=j \Rightarrow J_{i j}=\sum_{K \in i} \frac{1}{B_{i K}} \\
i \neq j \Rightarrow J_{i j}=-\frac{1}{B_{i j}}
\end{array} .\right.
$$

Uma vez obtida à solução para o sistema de equação, dado por (23), para a determinação do vetor de cargas, pode-se obter as vazões pela equação:

$$
Q=B^{-1} G Q_{0}-B^{-1} A_{12} H-B^{-1} A_{10} H_{0},
$$

que recai em uma estrutura de solução similar dada pelas equações(11) e (14). O processo de solução é análogo, ou seja, resolve-se o sistema de equações para a determinação das cargas, equação (23), e com os valores de carga obtidos, determina-se as vazões nos tubos por operações matriciais simples obtidas pela equação (25). 
Deve-se observar que a matriz inversa, que permite a solução do sistema de equações, equação (11 - modelo estático), depende de $G$, que é expresso pelas vazões nas tubulações, sendo portando calculada em cada iteração. No caso da equação (23 - modelo dinâmico) a matriz inversa se mantém constante durante todo o processo sendo, portanto, necessário calculá-la apenas uma vez, uma vez que B não está expresso em termos das vazões. Os vetores solução apresentam, em ambos os casos, grau semelhante de complexidade de cálculo. Esta observação pode ser importante na comparação entre os métodos, pois embora o tempo de convergência possa ser maior, o fato de não necessitar o recálculo da matriz inversa, caso o sistema seja demasiadamente grande, pode-se reduzir significativamente o tempo final de processamento.

Cabe aqui observar a diferença fundamental de convergência do método não inercial e o inercial proposto. No primeiro caso a convergência numérica é decorrente do processo de minimização de resíduos; no segundo caso trata-se de uma evolução no tempo (convergência no tempo) em que o fenômeno é descrito fisicamente a partir de uma condição inicial para uma outra condição qualquer.

Se o interesse é obter a condição estática, como faz o modelo estático empregado no EPANET, o modelo proposto também poderá ser empregado. Nesse caso pode-se abrir mão da precisão numérica, durante a condição transitória, para acelerar a convergência para o regime permanente, adotando-se valores de dt maiores. Cabe observar que o dt é a discretização numérica de uma equação diferencial e, portanto, para garantir sua adequação ao fenômeno físico este valor deve ser pequeno, confirmandoa representação da derivada. O uso de dt's grandes (dentro de certos limites, como serão observados em exemplos ilustrativos) não traduz corretamente a evolução do fenômeno físico, embora para os testes efetuados tenha-se obtido a convergência para o regime permanente final.

\section{ESTUDO DE CASOS}

Foram realizadas simulações para avaliar a eficácia do MDIR. A seguir são estudadas algumas topologias de sistemas hidráulicos para validar o modelo proposto.

\section{CASO 01}

Para estudar o efeito da inércia e a adequação do modelo numérico proposto, deve-se, primeiramente, comparar os resultados desse modelo com os resultados obtidos por solução analítica para um caso simples. Utilizou-se um sistema hidráulico composto de um reservatório, nível constante igual a $10 \mathrm{~m}$,

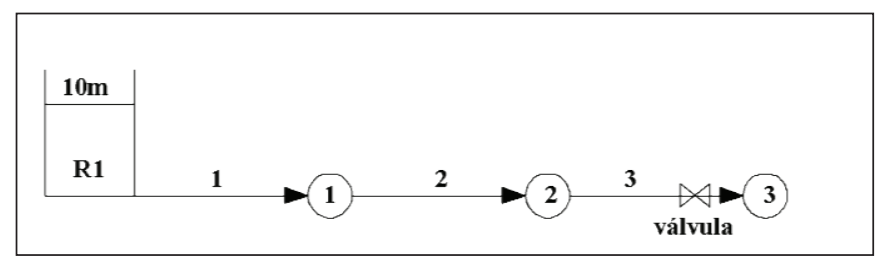

Figura 1 - Reservatório - tubo tubulação de $900 \mathrm{~m}$ de comprimento, diâmetro de 400mm e uma válvula localizada na saída da tubulação, como mostra a figura 1.

Para a utilização do MDIR necessita-se de pelo menos dois nós intermediários para se montar a matriz $J$ de 2X2. Logo, dois nós foram inseridos entre o reservatório (R1) e o nó de saída (nó 3), com espaçamentos iguais de 300m de comprimento. Foi realizada uma manobra de abertura instantânea da válvula. A tubulação, inicialmente, tem vazão nula, e durante a simulação o nível do reservatório foi mantido constante. A solução dinâmica da evolução da vazão ao longo do tempo, para este problema, é conhecida, na forma analítica, pela equação:

$$
Q=Q_{0} \frac{e^{5,29 \frac{t}{T_{r}}}-1}{e^{5,29 \frac{t}{T_{r}}}+1},
$$

onde :

$$
T_{r}=\frac{5,29 L Q_{0}}{2 g A H_{R}},
$$

sendo o tempo de estabelecimento do regime permanente final, $t_{\mathrm{O}}$ tempo de evolução transitória, $L$ comprimento do tubo, $H_{R}$ a altura do nível de água no reservatório e $Q_{0}$ a vazão final do regime permanente, o que permite avaliar o modelo numérico, como mostra a figura 2:

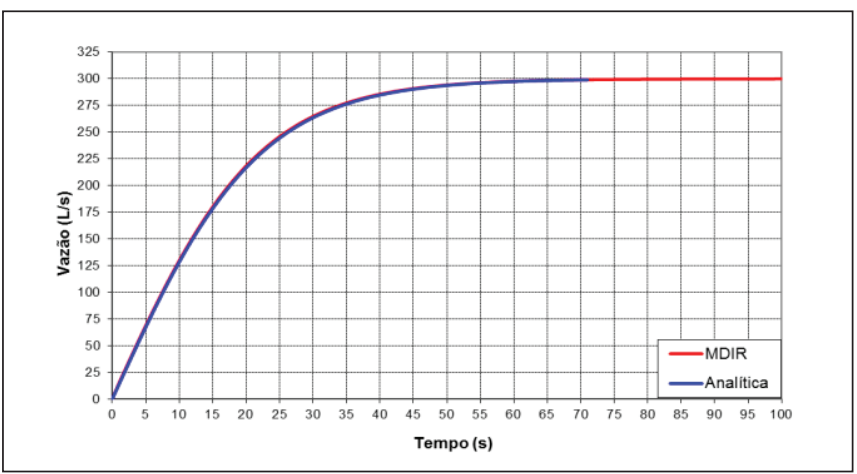

Figura 2 - Solução numérica x solução analítica

A mesma situação foi modelada no programa EPANET mostrando a diferença relativa ao efeito da inércia, como mostra a figura 3 :

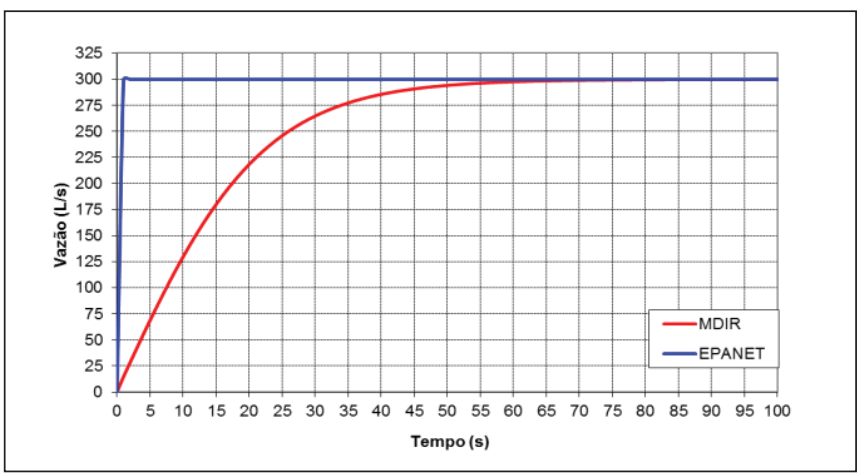

Figura 3 - MDIR x EPANET 
Nota-se que a busca do regime permanente pelo MDIR é mais realista, fisicamente. Como já foi abordado, para a obtenção da condição estática, como faz o modo estático empregado no EPANET, o modelo proposto também poderá ser empregado. Nesse caso, pode-se abrir mão da precisão numérica durante a condição transitória para acelerar aconvergência para o regime permanente, adotando-se valores de dt maiores como mostra a figura 4:

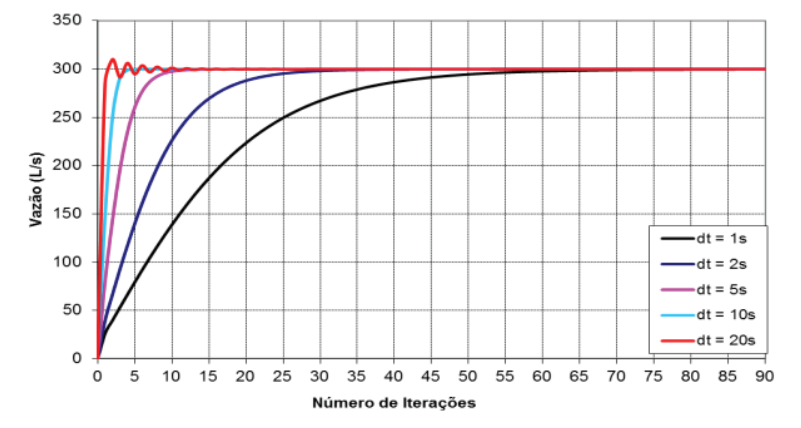

Figura 4 - Convergência numérica para diferentes valores de dt

O uso de dt's grandes não traduz corretamente a evolução do fenômeno físico, embora para os testes efetuados tenhase obtido a convergência para o regime permanente final. Nos testes realizados, valores de dt a partir de $20 \mathrm{~s}$, configuraram má representação intermediária do fenômeno.

\section{CASO 02}

A segunda topologia analisada foi um sistema hidráulico composto de dois reservatórios ligados por um tubo como mostra a figura 5 a seguir:

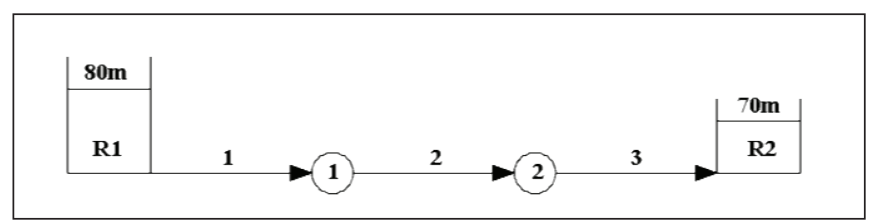

Figura 5 - Reservatório - tubo - reservatório

Para se utilizar o MDIR, novamente, necessitou-se de dois nós intermediários para poder montar a matriz $J$. O sistema hidráulico é análogo ao sistema da figura 1, onde inseriu-se um reservatório (R2) no lugar do nó 3. Mais uma vez, o tubo é de $900 \mathrm{~m}$ de comprimento, com espaçamentos iguais de $300 \mathrm{~m}$ e diâmetro do tubo de $400 \mathrm{~mm}$.

Para a simulação do regime transitório, uma demanda de $100 \mathrm{~L} / \mathrm{s}$ foi inserida durante 150 s no nó 1 . Nas figuras. 6 a 11, os valores de carga hidráulica e vazão são apresentados, calculados pelos dois modelos. Observe que a figura7 é uma continuação da figura 6 , assim como figura 8 é uma continuação da figura 9.

Note que o EPANET, uma vez que não considera o efeito da inércia do escoamento, simplesmente atualiza os dados de carga hidráulica e vazão para a nova condição imposta. O mesmo não acontece com os resultados do MDIR. A figura 6 , por exemplo, mostra o pico de pressão no nó 1 , quando uma demanda de $100 \mathrm{~L} / \mathrm{s}$, foi aí inserida.

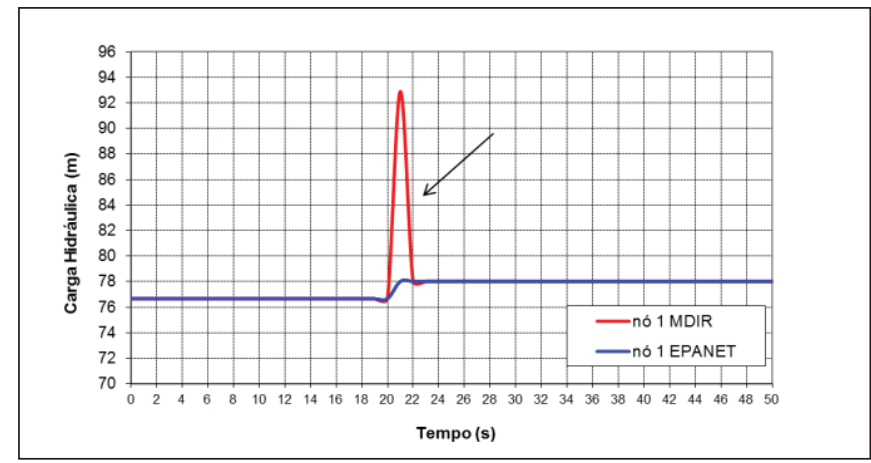

Figura 6 - Valores de Carga Hidráulica no nó 1 calculada pelos dois Modelos

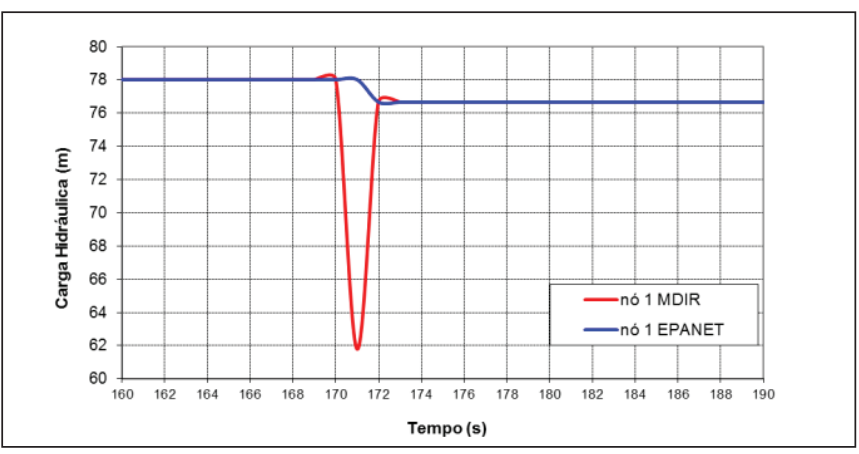

Figura 7- Valores de Carga Hidráulica no nó 1 calculada pelos dois Modelos

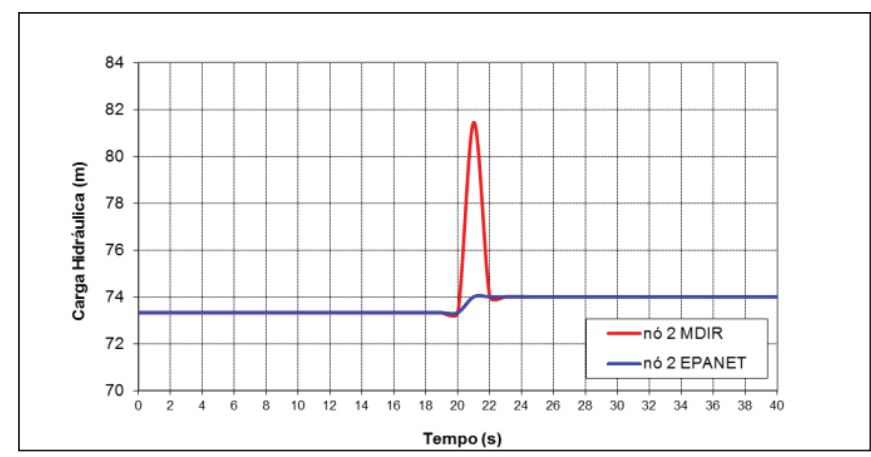

Figura 8 - Valores de Carga Hidráulica no nó 2 calculada pelos dois Modelos

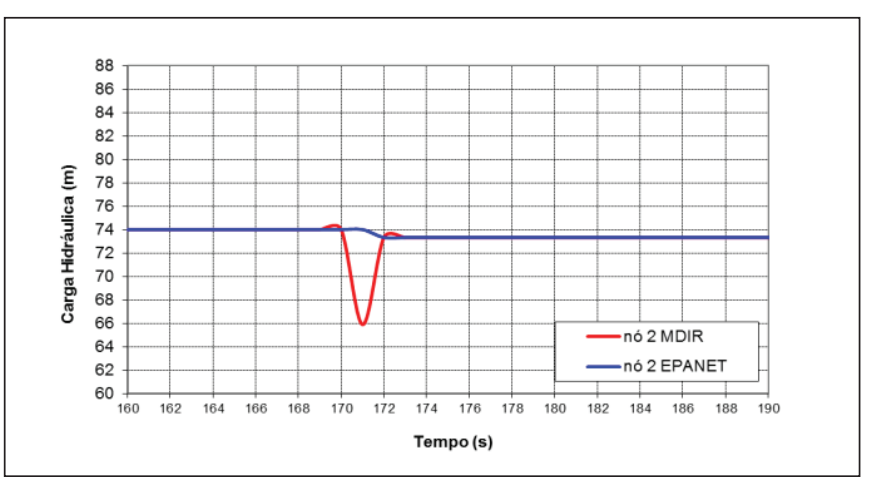

Figura 9 - Valores de Carga Hidráulica no nó 2 calculada pelos dois Modelos 


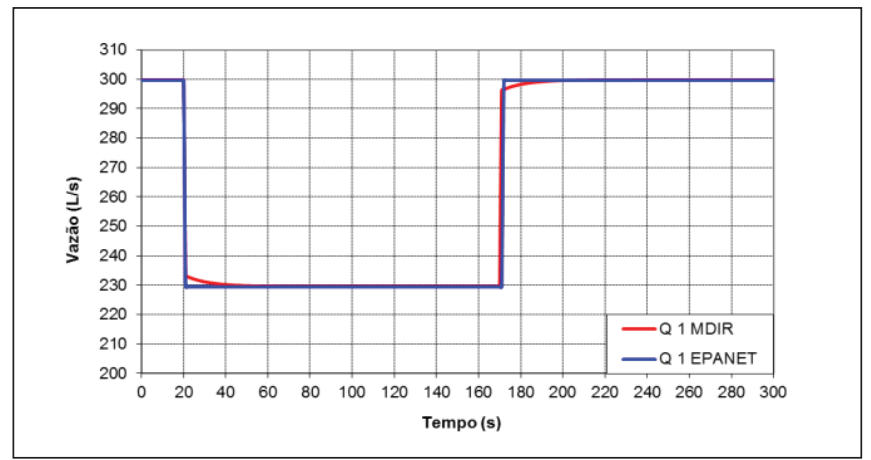

Figura 10 - Valores de Vazão no tubo1 calculada pelos dois Modelos

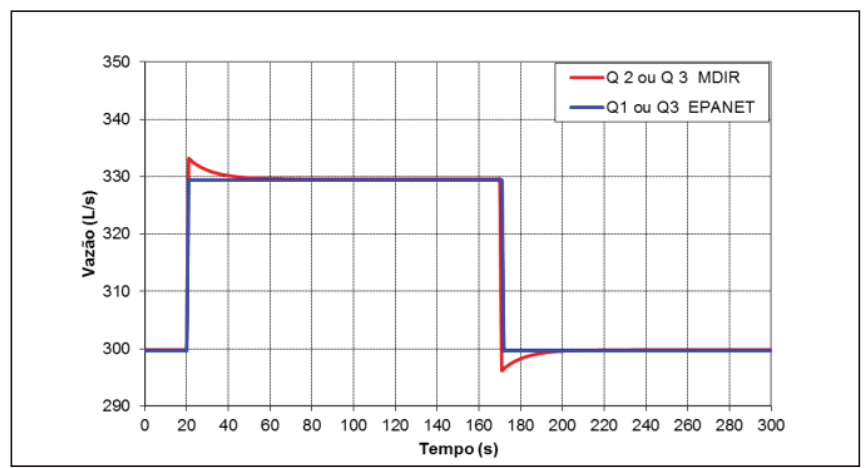

Figura 11 - Valores de Vazão nos tubos 2 e 3 calculada pelos dois Modelos

O efeito de inércia obtido pelo MDIR é melhor observado através da seguinte simulação: Os reservatórios foram criados em diferentes níveis e com uma secção circular de diâmetro de $3 \mathrm{~m}$. O tubo é de 90 metros de comprimento, novamente dividido pelosnós 1 e 2, ou seja, em três partes iguais de $30 \mathrm{~m}$. $\mathrm{O}$ diâmetro de cada secção foi alterado para $600 \mathrm{~mm}$. Todas as simulações começaram com o estado estacionário, estando osreservatórios R1 e R2 com cargas hidráulicas iniciais de 80 $\mathrm{m}$ e $70 \mathrm{~m}$, respectivamente. A discretização temporal foi $\mathrm{dt}=1$ s.Os resultados obtidos pelo MDIR são comparados com aqueles do EPANET, para a carga hidráulica, apresentados nas figuras 12 e 13, o que evidencia o efeito da inércia na representação fiel do fenômeno físico.

Note que esta simulação, mostra o efeito de inércia de drenagem, também chamada de pêndulo hidráulico, em que os níveis dos dois reservatórios de água irregulares são equalizados para alcançar o último regime permanente. Quando tubos com diâmetros menores são adotados, para qualquer comprimento conhecido, com consequente inércia menor e perda de carga maior, a dissipação da onda de pressão é maior e os resultados encontrados pelo MDIR e EPANET aproximam-se. No entanto, como o diâmetro do tubo aumenta e é muito mais evidente, a dissipação de onda é menor, como pode ser visto nos valores apresentados nas figuras 12 e 13.

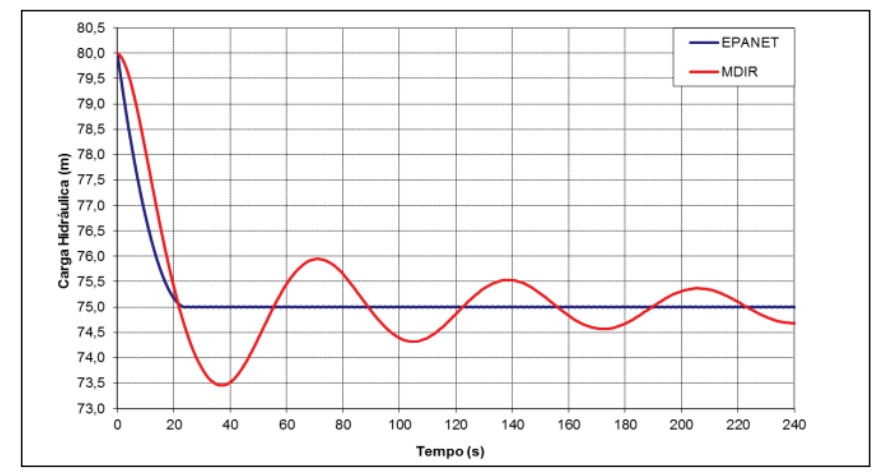

Figura 12 - Carga Hidráulica no Reservatório R1

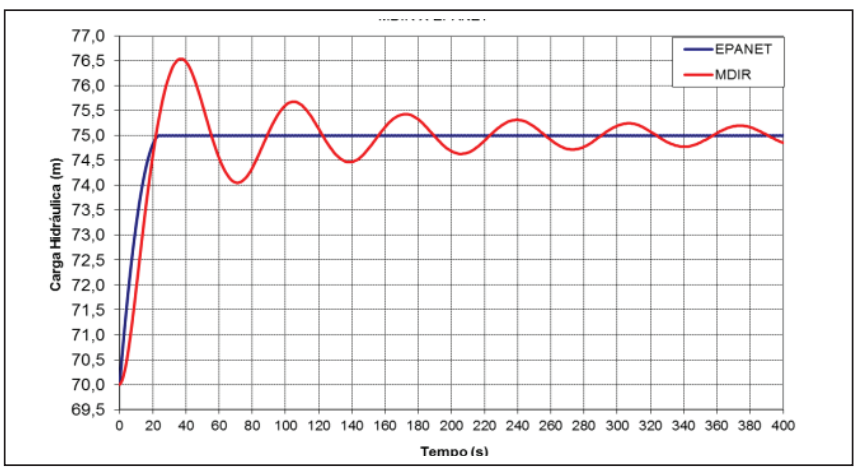

Figura 13 - Carga Hidráulica no Reservatório R2

\section{CASO 03}

A próxima rede em estudo, figura 14, foi proposta por Luvizotto Jr e Anjo (2004). Ela é composta por 21 tubos e 14 nós, sendo um de carga fixa (nó 14). O nó 14 corresponde ao reservatório de nível constante com 100m de carga hidráulica.

Os dados referentes à rede hidráulica estão apresentados nas tabelas 1 e 2 , a seguir:

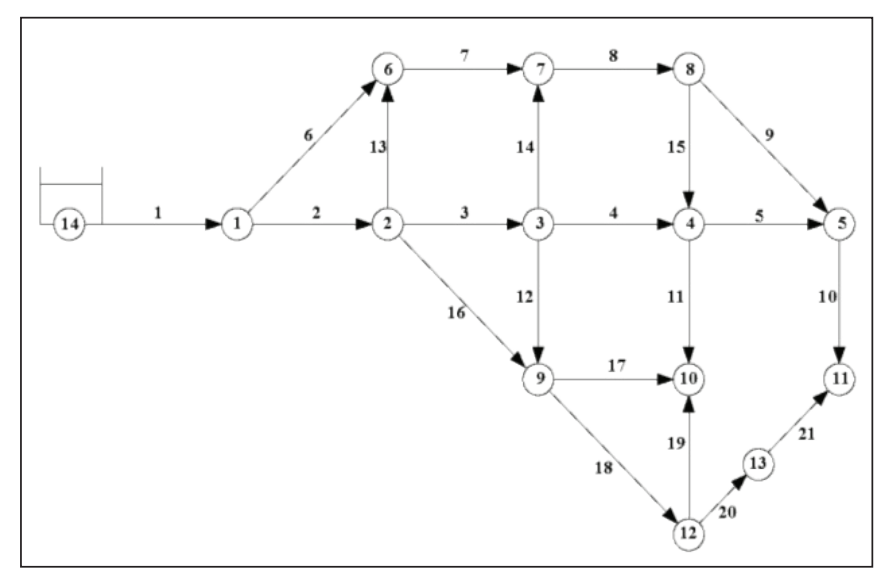

Figura 14 - Rede hidráulica mais complexa 
Tabela 1 - Valores referentes aos tubos da rede hidráulica

\begin{tabular}{c|c|c|c|c|c}
\hline $\begin{array}{c}\text { Tub } \\
\text { o }\end{array}$ & Nó & Nó & e (m) & L (m) & D (m) \\
\hline 1 & 14 & 1 & 0,0001 & 500 & 0,4 \\
\hline 2 & 1 & 2 & 0,0001 & 400 & 0,4 \\
\hline 3 & 2 & 3 & 0,0001 & 300 & 0,4 \\
\hline 4 & 3 & 4 & 0,0001 & 300 & 0,2 \\
\hline 5 & 4 & 5 & 0,0001 & 300 & 0,2 \\
\hline 6 & 1 & 6 & 0,0001 & 400 & 0,3 \\
\hline 7 & 6 & 7 & 0,0001 & 300 & 0,3 \\
\hline 8 & 7 & 8 & 0,0001 & 300 & 0,3 \\
\hline 9 & 8 & 5 & 0,0001 & 350 & 0,3 \\
\hline 10 & 5 & 11 & 0,0001 & 300 & 0,3 \\
\hline 11 & 4 & 10 & 0,0001 & 300 & 0,2 \\
\hline 12 & 3 & 9 & 0,0001 & 300 & 0,2 \\
\hline 13 & 2 & 6 & 0,0001 & 300 & 0,3 \\
\hline 14 & 3 & 7 & 0,0001 & 300 & 0,2 \\
\hline 15 & 8 & 4 & 0,0001 & 300 & 0,2 \\
\hline 16 & 2 & 9 & 0,0001 & 350 & 0,3 \\
\hline 17 & 9 & 10 & 0,0001 & 300 & 0,2 \\
\hline 18 & 9 & 12 & 0,0001 & 350 & 0,3 \\
\hline 19 & 12 & 10 & 0,0001 & 300 & 0,2 \\
\hline 20 & 12 & 13 & 0,0001 & 150 & 0,3 \\
\hline 21 & 12 & 11 & 0,0001 & 200 & 0,3 \\
\hline & & & & &
\end{tabular}

Tabela 2 - Valores referentes aos nós da rede hidráulica

\begin{tabular}{c|c|c}
\hline Nó & $\begin{array}{c}\text { Cota } \\
(\mathrm{m})\end{array}$ & $\begin{array}{c}\text { Demand } \\
\mathrm{a}\left(\mathrm{m}^{3} / \mathrm{s}\right)\end{array}$ \\
\hline 1 & 0,00 & 0,000 \\
\hline 2 & 0,00 & 0,015 \\
\hline 3 & 0,00 & 0,015 \\
\hline 4 & 0,00 & 0,015 \\
\hline 5 & 0,00 & 0,015 \\
\hline 6 & 0,00 & 0,025 \\
\hline 7 & 0,00 & 0,015 \\
\hline 8 & 0,00 & 0,015 \\
\hline 9 & 0,00 & 0,015 \\
\hline 10 & 0,00 & 0,025 \\
\hline 11 & 0,00 & 0,020 \\
\hline 12 & 0,00 & 0,015 \\
\hline 13 & 0,00 & 0,000 \\
\hline
\end{tabular}

A primeira simulação foi realizada para determinar, a partir de um dado instante (fluxo constante inicial estabelecido), os resultados de um fluxo de entrada no nó (13) sobre a função da cabeça deste nó, usando:

$$
q=0,00007 H^{1 / 2} .
$$

A entrada desse escoamento corresponde a simulação de uma saída no tubo 20, e os resultados calculados pelo MDIR e EPANET, para a carga no nó 1, estão apresentadas na figura 15.

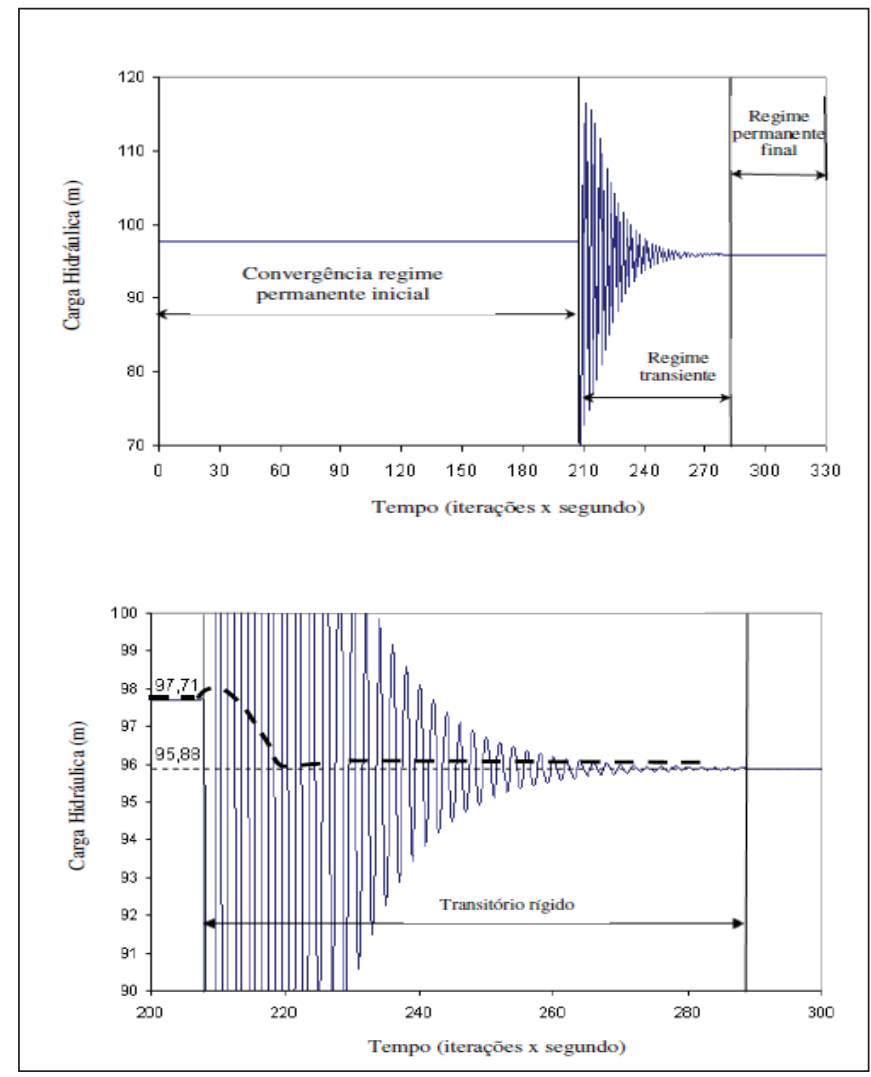

Figura 15 - Convergência da Carga Hidráulica calculada pelo MDIR e EPANET

Observa-se, na figura 15, que a variação na carga hidráulica calculada pelo MDIR é significativa, enquanto o EPANET simplesmente atualiza os valores para a nova situação imposta pelo vazamento. O MDIR faz pesquisas físicas, mais próximas da realidade, enquanto o EPANET realiza uma pesquisa numérica, até se adaptar à nova situação.

\section{CONCLUSÕES}

Os resultados obtidos permitem concluir sobre a possibilidade de emprego do modelo proposto adicionando-o a estrutura do programa EPANET, ampliando sua análise para o caso de transitórios lentos produzidos, por exemplo, pela alteração dos consumos setoriais. A formulação proposta seguiu a mesma adotada pelo método gradiente, produzindo poucas alterações no algoritmo original (essencialmente na matriz do sistema) para incorporar as vantagens da análise dinâmica. Em outras palavras, a adequação do modelo do EPANET a um modelo inercial rígido é simples, com pouca mudança em sua estrutura principal. Tal modificação pode proporcionar uma melhor adequação às simulações em período extensivo como ficou demonstrado nos estudos de casos hipotéticos apresentados. Uma vantagem do modelo proposto, além da simulação dinâmica mais coerente com a dinâmica do escoamento, está no fato de se calcular apenas uma vez a equação (19), uma vez que o termo não está expresso em termos das vazões. Isso significa 
que a matriz do sistema de equações é calculada uma única vez durante a evolução da simulação no tempo. Por outro lado, no modelo original do EPANET é necessário refazer a matriz do sistema de equações a cada passo de cálculo.

A convergência no tempo do MDIR é mais morosa, porém, o fato de não necessitar do recalculo da matriz do sistema, isso pode ser uma vantagem se o sistema for demasiadamente grande, podendo reduzir significativamente o tempo final de processamento do modelo dinâmico.

O fato de o MDIR levar em consideração o efeito da inércia em sua estrutura de cálculo, as simulações que foram realizadas nas diversas topologias analisadas mostraram-se mais adequadas à realidade para a simulação em período extensivo. Vale ressaltar que esse trabalho teve como intuito analisar esse efeito da inércia quando comparado com a análise em período extensivo. Como já descrito anteriormente, investigações parecidas entre tais modelos foram realizadas por Iglesias (2004), justificando a importância desse estudo. Neste sentido, Ahmed e Lansey (1999), simulando um sistema hidráulico composto por 21 tubos e 13 nós, e um reservatório com carga fixa, semelhante ao caso 3 deste trabalho, destacaram a necessidade de intensificar a aplicação do modelo computacional dinâmico, por eles desenvolvido, com base nas equações hidrodinâmicas completas. Os resultados obtidos pelo modelo foram comparados com os da simulação em período extensivo do programa Kypipe, demonstrando diferenças, tanto em direção, quanto em magnitude, do efeito da inércia. A intensificação das aplicações, sugerida pelos autores, visa identificar se estas diferenças observadas, são significativas em sistemas gerais.

O presente estudo, longe de finalizar a investigação sobre a possibilidade da incorporação do modelo rígido ao programa EPANET, abre caminho para outras investigações que permitam definir de uma forma simples a fronteira entre modelos.

Outras questões que surgem são relativas à possibilidade de um equacionamento dinâmico de alguns elementos, permitindo, por exemplo, a análise dinâmica do comportamento de uma bomba com mudança de rotação ou o efeito dinâmico de uma válvula de controle. Considerando os resultados específicos deste trabalho, as conclusões do trabalho de Ahmed e Lansey (1999), dentro das várias situações passíveis de análise dos modelos, a continuidade de testes computacionais e explorações matemáticas, conduzirão, cada vez mais, a resultados realistas, em um grau expressivo de confiabilidade, no que tange ao suporte da modelagem para a operação de sistemas hidráulicos.

\section{SIMBOLOGIA}

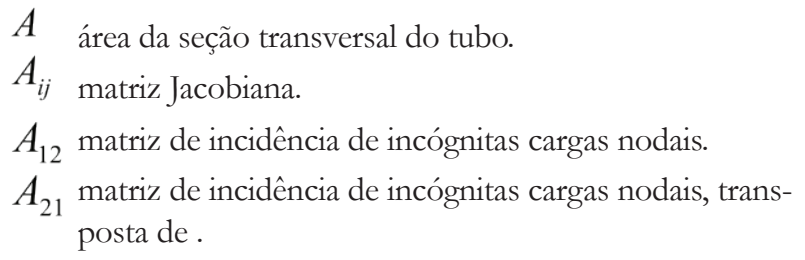

$A_{10}$ matriz de incidência de nós com carga fixa.

$A_{11}$ matriz dos coeficientes de energia.

$a$ celeridade.

$B$ matriz diagonal quadrada de ordem np.

$C$ concentração de um componente.

$D$ diâmetro do tubo.

$D_{s}$ coeficiente de dispersão.

e espessura da tubulação

$f(Q)$ lei que expressa a variação de carga nos tubos.

$G$ matriz diagonal quadrada

$g$ aceleração da gravidade.

$H$ carga piezométrica no nó.

$H_{0}$ nó com carga hidráulica fixa ou conhecida.

$H_{1}$ cargapiezométrica no nó de montante.

$H_{2}$ cargapiezométrica no nó de jusante.

$\Delta H$ vetor de diferença de carga.

$J$ matriz jacobiana.

$L$ comprimento do tubo.

$Q$ descarga em volume nos tubos.

$q$ demanda no nó.

$R$ coeficiente de perda de carga.

$t$ tempo.

$\beta$ termo de inércia.

$\beta_{i i}$ matriz diagonal quadrada de ordem

\section{REFERÊNCIAS}

AHMED, I.; LANSEY, K. E. Analysis of unsteady flow in networks using a Gradient algorithm based method. In: ANNUAL WATER RESOURCES PLANNING AND MANAGEMENT CONFERENCE, ASCE, 26., Tempe, Arizona, EUA, 1999. Anais... Tempe, Arizona, EUA: ASCE, 1999.

CABRERA, E.; GARCIA-SERRA, J.; IGLESIAS, P. L. Modelización de redes de distribución de água. Desde el régimen permanente hasta el golpe de aríete, "Mejora del rendimiento y de la fiabilidad em sistemas de distribución de água". Espanha: Aguas de Valencia S.A. y U.D .Mecánica de Fluidos U.P.V., 1994.

IGLESIAS, P. L.; SOLANO, F. J. M.; SEBASTIÁN, J. I.; MIQUEL, V.S. F. Frontera de separación entre el modelo rígido y la simulacíon en periodo extendido en sistemas hidráulicos a presión. In: CONGRESO LATINOAMERICANO DE HIDRÁULICA, 1., São Pedro, São Paulo, 2004. Anais... São Pedro: [s.n.], 2004.

ISLAM, M. R.; CHAUDHRY, M. H. Modeling of constituent transport in unstead flows in pipe networks. Journal of Hydraulic, v. 124, n. 11, p. 1115-1124, Nov. 1998. 
RBRH vol. 20 n.2 Porto Alegre abr./jun. 2015 p. 533 - 542

LUVIZOTTTO Jr, E.; ANJO, L. F. R. S. Utilizando a estrutura do método gradiente na elaboração de um modelo para analisar transitórios lentos. CONGRESO LATINOAMERICANO DE HIDRÁUliCA, 21., São Pedro, São Paulo, 2004. Anais... São Pedro: [s.n.], 2004.

ROSSMAN, L. A. EPANET - Users manual. Cincinnati, Ohio: National Risk Management Research Laboratory, Office of Research and Development, U. S. Environmental Protection Agency, 1993.

TODINI, E.; PILAT, S. A gradient algorithm for the analysis of pipe networks. In: COUBECK, K. B.; ORR, C.H. Computer applications in water supply: vol. 1 - System analysis and simulation. New Jersey: John Willey, 1988. p.1-20.

WYLIE, E. B.; STREETER, V. L. Fluid transients in systems. Englewood Cliffs, USA: Prentice Hall, 1993. 